\title{
CAMPUS NUMERIQUE - CAMPUS VIRTUEL : QUEL PUBLIC ?
}

\author{
Jean GRIEU ${ }^{1}$, Florence LECROQ ${ }^{2}$ \\ ${ }^{1}$ Maître de Conférences, Département Gestion des Entreprises et des Administration, IUT du Havre, Place Robert \\ Schuman, 76610 Le Havre. \\ Email : jean.grieu@univ-lehavre.fr \\ ${ }^{2}$ Maître de Conférences, Département Génie Electrique et Informatique Industrielle, IUT du Havre, Place Robert \\ Schuman, 76610 Le Havre. \\ Email : florence.lecroq@univ-lehavre.fr
}

\begin{abstract}
RESUME :
Grâce au langage SCOL, une représentation 3D du monde réel est possible. A la différence d'un campus numérique, cette représentation devient un campus virtuel dans lequel les apprenants peuvent évoluer avec des avatars. Ils ont alors une notion d'appartenance à ce monde puisqu'ils participent à sa création par leurs présences. Les outils de formation sont disponibles dans les différentes pièces du campus virtuel. Parallèlement à la création de ce monde virtuel, une étude a été menée auprès d'étudiants pour connaître leurs attentes sur les outils disponibles dans ces nouveaux campus. Cet outil prometteur semble correspondre aux attentes et aux habitudes de vie de nos étudiants.
\end{abstract}

Mots clés : Environnement 3D, formation à distance, campus numérique, campus virtuel, langage SCOL

\section{INTRODUCTION}

Il existe déjà un grand nombre de plates-formes pédagogiques pour assister la e-formation [1]. Malgré l'attrait des nouvelles technologies, ces nouvelles formations connaissent déjà une difficulté majeure par le taux d'abandon des apprenants. Selon Carr [2], le taux d'abandon est de $10 \%$ à $20 \%$ plus élevé que dans les formations en mode présentiel. Le choix de la plate-forme pédagogique est donc fondamental. Il existe une différence de public entre les utilisateurs d'un campus numérique et ceux d'un campus virtuel qu'il ne faut pas négliger. C'est pour essayer de comprendre les attentes de nos étudiants que nous avons effectué un sondage auprès de 108 étudiants de $\mathrm{GEA}^{1}$ (2ième année) et de 105 étudiants $\mathrm{GEII}^{2}\left(1^{\text {ère }}\right.$ et $2^{\text {ième }}$ année), soit un effectif global de 213 étudiants. A partir de cette étude, nous espérons approcher leurs habitudes de vie, évaluer leur équipement informatique, et surtout découvrir leur point d'attraits de l'Internet. Enfin, nous appuyant sur des travaux déjà réalisés concernant le bien fondé de l'utilisation d'un environnement 3D dans le e-learning [3], nous présenterons une plate-forme de type $\mathrm{SCOL}^{3}$.

\footnotetext{
${ }^{1}$ GEA : Gestion des Entreprises et des Administration.

${ }^{2}$ GEII : Génie Electrique et Informatique Industrielle.

${ }^{3}$ SCOL : langage open source de conception de monde 3D, créé par Sylvain HUET.
}

Il est à noter que l'université du Havre est équipée depuis le début de l'année universitaire 2004-2005 d'un campus numérique (Claroline) ${ }^{4}$.

\section{LES RESULTATS DU SONDAGE SUR LES ETUDIANTS}

L'ensemble de l'étude nous donne un public essentiellement masculin (65\%), les garçons étant majoritaires chez les GEII $(96 \%$ de garçons) et minoritaires chez les GEA (65\% de filles). Les GEII jouent plus sur les jeux 3D (oui : 90\%) que les GEA (oui : $76 \%$ ), ont joués en réseau (oui : $55 \%$ ) et pas les GEA (non : 81\%), mais nos étudiants se rejoignent dans la possession d'une console $(85 \%$ des GEII ont une console, $80 \%$ des GEA en ont une). Beaucoup d'étudiants $(68 \%)$ possèdent un accès Internet personnel, qui est de bonne qualité $(50 \%$ sont sur l'ADSL). Cette connexion est souvent utilisée (chaque jour : 37\%, de 1 à plusieurs fois par semaine : 33\%), pour un temps moyen de connexion important à chaque fois $(29 \%$ entre 1 et 3 heures, $17 \%$ supérieur à 3 heures, 26\% pour 1 heure). Ils se servent de cette connexion :

* moyennement (33\%) pour le travail

* de beaucoup (34\%) à moyennement $(28 \%)$ pour le divertissement

* de moyennement $(30 \%)$ à très peu $(23 \%)$ pour la culture et l'actualité

\footnotetext{
${ }^{4}$ Claroline : plate-forme pédagogique, disponible en open source, Université de Louvain.
} 
* de beaucoup (31\%) à moyennement (23\%) pour la communication.

Il faut noter ici une différence d'utilisation de la connexion à Internet entre les étudiants de GEII et ceux de GEA :

- les GEA l'utilisent de moyennement (35\%) à beaucoup (31\%) pour le travail, alors que les GEII l'utilisent moyennement $(30 \%)$ et très peu $(30 \%)$ pour cela.

* Les GEA l'utilisent de moyennement (32\%) à beaucoup (29\%) pour le divertissement, alors que les GEII l'utilisent de beaucoup (39\%) à moyennement $(24 \%)$.

* Les GEII l'utilisent un peu plus pour la communication que les GEA $(35 \%$ de «beaucoup » chez les GEII, contre 26\% de « beaucoup » chez les GEA).

La communication se fait essentiellement par les messageries instantanées (62\% les utilisent) et par le biais des Emails (74\% en envoient). Pour nos étudiants, les qualités d'un «bon site web» sont d'abord la rapidité, puis le contenu et enfin la clarté. Ils ont majoritairement (69\%) visité le site de leur département. Quant à la plate-forme pédagogique Euréka (Claroline), une grande majorité $(72 \%)$ ne la connaît pas, et encore plus $(85 \%)$ ne l'ont pas utilisée. Selon eux, une plate-forme pédagogique sur Internet doit être un complément au cours traditionnel (85\%), plutôt qu'une solution complète et suffisante $(9 \%)$. Dans l'acquisition des connaissances, ils estiment la présence du professeur indispensable $(62 \%)$, voir parfois utile (34\%). Selon eux, les nouvelles technologies isolent les individus plutôt qu'elles ne les rapprochent, et améliorent la communication plutôt qu'elles ne la dégradent.

Dans leurs souhaits d'un campus numérique idéal, nos étudiants aimeraient :

t) d'abord avoir leur emploi du temps,

16 des compléments au cours traditionnel,

t des exercices et des anciens devoirs surveillés avec des corrigés,

* une assistance en ligne avec les professeurs et un espace de communication avec eux,

4 un site rapide et simple

* et un accès personnel aux notes.

Ils souhaiteraient aussi un espace pour des petites annonces ainsi qu'un espace d'évaluation, et enfin, pouvoir accéder à plusieurs en même temps sur le site.

De ce sondage, nous pouvons tirer quelques conclusions :

* Le campus numérique doit être considéré comme une aide optionnelle, mais nos étudiants ne veulent pas abandonner les cours en présentiel. Cette université virtuelle ne changera rien à l'absentéisme des cours. En effet, ceux qui ont répondu à ce questionnaire étaient présents en cours (108 étudiants GEA sur 123 inscrits et 105 étudiants GEII sur 164 inscrits), alors que l'année est fortement avancée. Nous ne connaissons pas les raisons des absents...

* Il ressort également de cette étude une forte demande de communication entre les étudiants et les professeurs. Nos impétrants veulent aussi se rencontrer à plusieurs sur le site, recherchant ainsi plus d'interactivité et de communication synchrone.

* La plate-forme pédagogique Euréka (Claroline) ne les attire pas, et ce n'est pas à cause d'un problème technique, puisqu'ils ont pour beaucoup un accès au réseau par l'ADSL. Le public des GEA pourrait correspondre à une plateforme numérique, alors que le public des GEII semble parfait pour un campus virtuel mais pas numérique.

\section{UNE SOLUTION}

Aux vues des résultats de ce sondage, le choix d'un campus virtuel plutôt que d'une plate-forme pédagogique traditionnelle s'est naturellement fait pour les étudiants de GEII. De plus, ils connaissent parfaitement le fonctionnement des jeux $3 \mathrm{D}$ en réseau. Le sentiment d'appartenance au monde virtuel par l'interface des avatars devrait les séduirent. Pour répondre à leurs attentes, nous avons donc réalisé un campus virtuel, avec la technologie SCOL. Le choix de ce langage de programmation s'est imposé avec notre cahier des charges: site accessible rapidement par n'importe quel outil informatique ${ }^{5}$, gestion de base de données $\mathrm{SQL}^{6}$, visioconférence, création d'un espace 3D.

Grâce au langage SCOL, nous avons recréé un environnement familier pour la première salle d'accueil (figure $\mathrm{n}^{\circ} 1$ ).

\footnotetext{
${ }^{5}$ Outil informatique : machine modeste ou puissante, Windows ou Linux, combo casque micro ou pas, simple modem ou haut débit, carte graphique simple ou performante, etc.

${ }^{6}$ SQL : (Structured Query Language), langage de définition, de manipulation et de contrôle de données.
} 


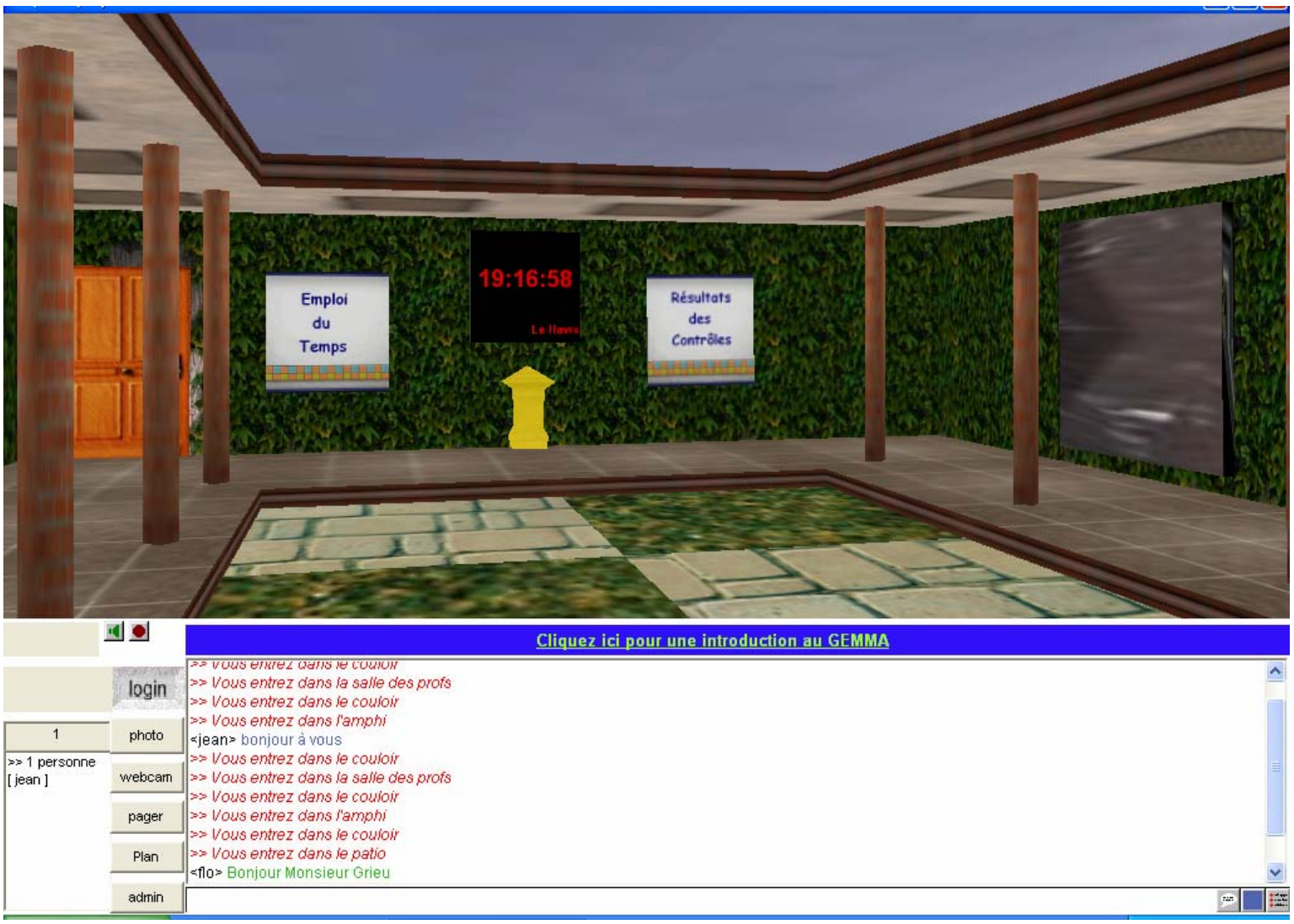

figure 1 : L'entrée du campus virtuel

Ce patio est une copie de l'existant (Département GEII IUT du Havre), avec des outils particuliers :

+ Dans la fenêtre principale :

une boîte aux lettres pour envoyer des Emails,

- un tableau donnant accès aux emplois du temps,

un tableau accédant aux notes,

un tableau de mode d'emploi du système,

- une horloge (pour être à l'heure !)

- et une porte pour accéder au reste du campus.

* Dans la partie basse de la fenêtre :

- A gauche, on retrouve le nombre et les noms des personnes connectées,

- Un certain nombre de fonctions pour entrer sur le site, choisir son avatar ${ }^{7}$, une fonction pour du « chat $^{8} »$ privé entre deux connectés, un accès au plan général du site.

\footnotetext{
${ }^{7}$ avatar : Personnage numérique qui représente dans le cyberespace chacune des personnes se rencontrant pour bavarder en direct, de façon interactive, dans une réunion sociale virtuelle. Un avatar est en quelque sorte notre alter ego dans le cyberespace [4].

${ }^{8}$ chat : De l'anglais bavarder. Échange de messages textuels sur le Web, entre deux (chat privé) ou plusieurs (chat public) individus connectés. Il s'agit
}

- Sous le bandeau bleu, qui est un menu déroulant donnant les informations de dernière minute, une zone décrivant les déplacements des différents intervenants dans les différentes pièces, ainsi que les « chats » publics.

La figure suivante (figure $\mathrm{n}^{\circ} 2$ ), représente la salle de cours, dans laquelle il est possible d'effectuer un cours synchrone avec un affichage sur grand écran (partie droite de l'image). Un avatar est positionné sur le premier rang de l'amphithéâtre.

d'un lieu de conversation en temps réel (synchrone), par opposition au forum qui est un lieu d'échange en temps différé (asynchrone) [5]. 


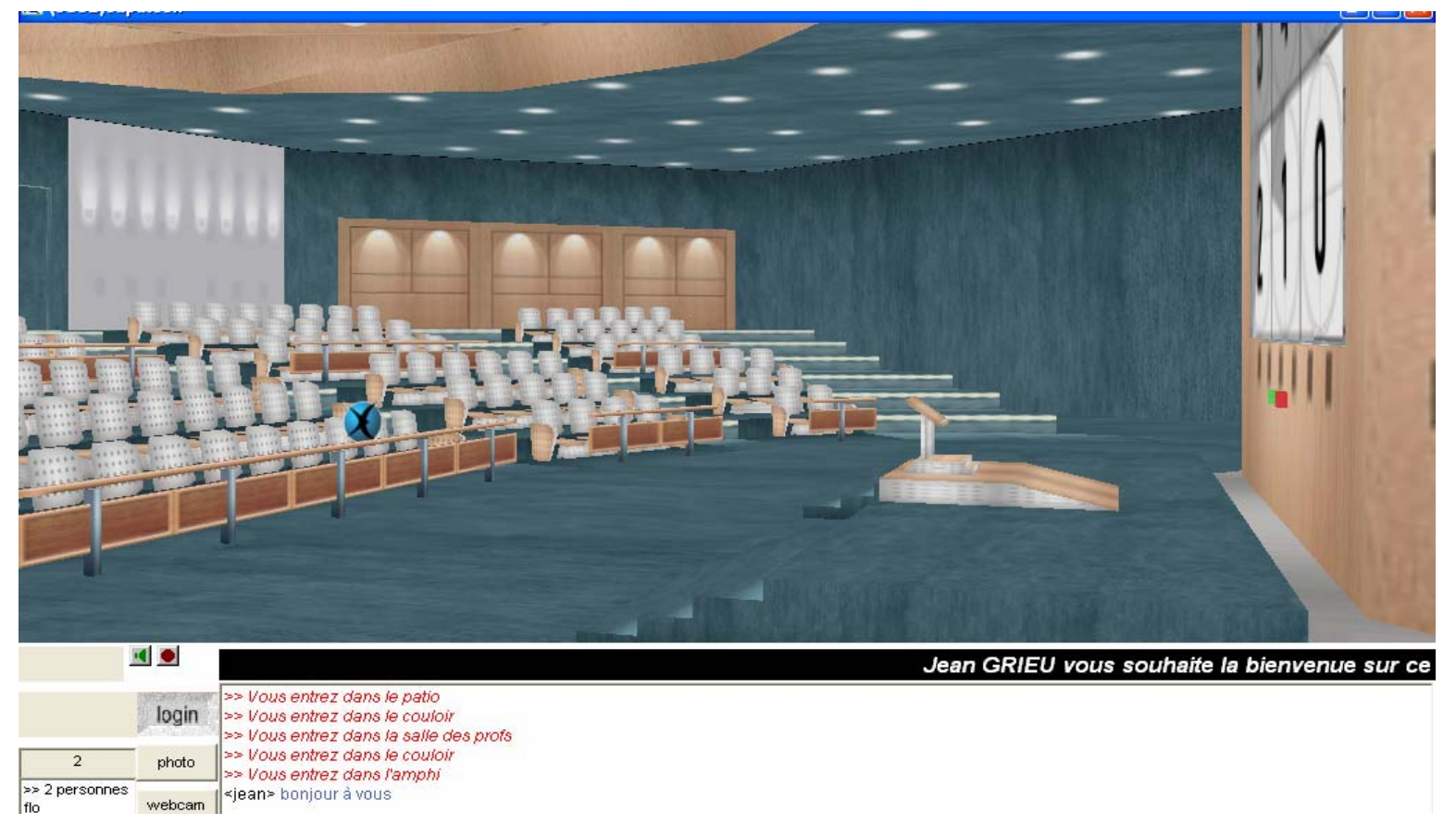

figure 2 : L'amphithéâtre avec un avatar au premier rang

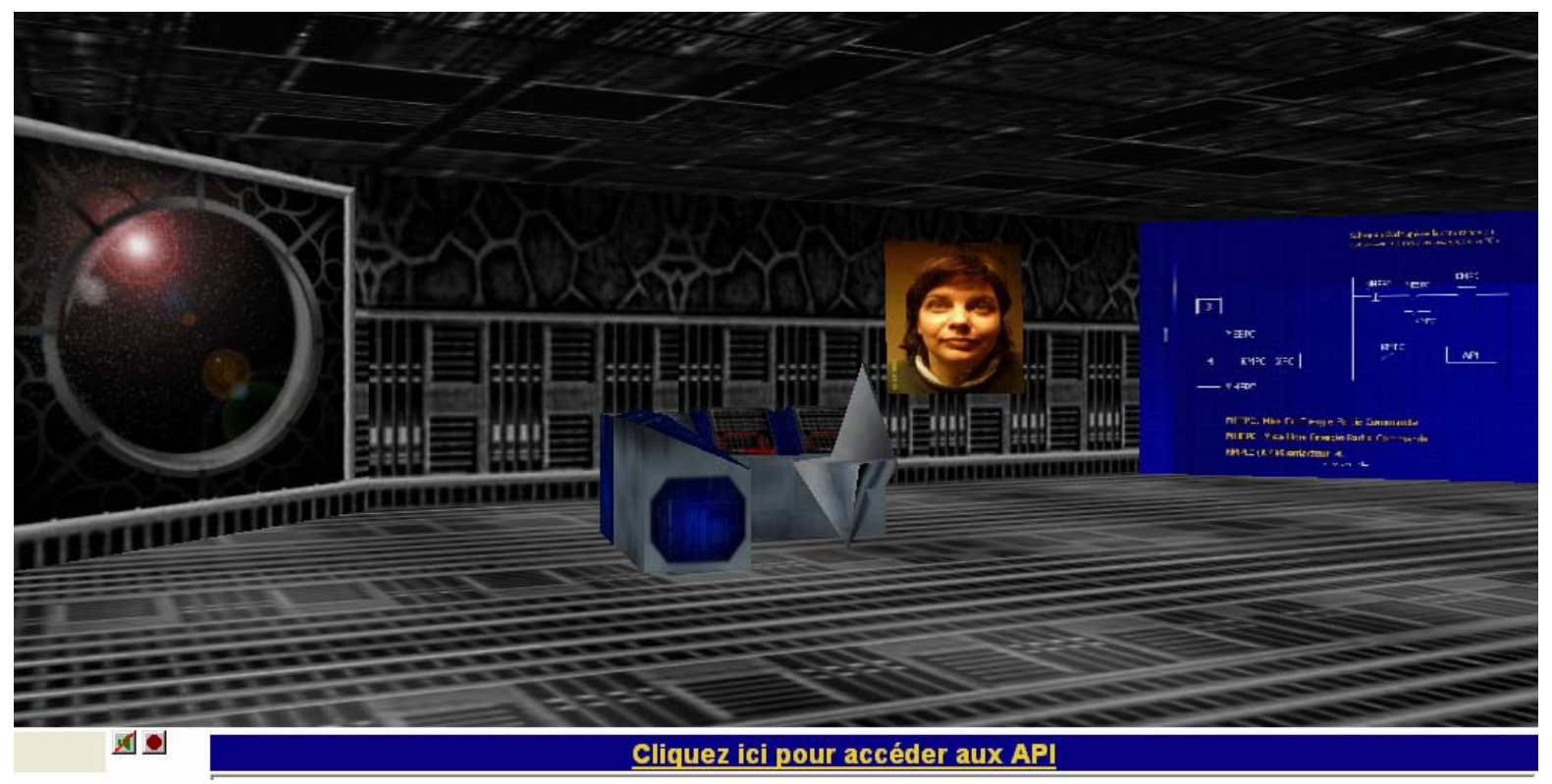

figure 3 : La salle des professeurs, avec une photo pour un avatar présent, et le tableau qui permet le partage synchrone de données (ici, du texte et un graphique sur fond bleu).

Une salle des professeurs (figure $n^{\circ} 3$ ) est aussi accessible à tous les visiteurs. Ici, un tableau blanc permet le partage synchrone d'une fenêtre graphique et textuelle à l'intérieur de laquelle tous les utilisateurs peuvent interagir simultanément. Cette fonction autorise le partage de documents et la possibilité d'élaborer des documents en temps réel qui seront visionnés par les apprenants et modifiables par chacun des participants. 


\section{Conclusion}

En 2005, les attentes de nos étudiants vis-à-vis des outils d'apprentissage ne sont plus les mêmes que celles qui pouvaient exister il y a dix ans. L'usage des outils actuels de communication et d'accès à l'information doit nous amener à reconsidérer notre démarche de conception des plates-formes de formation à distance. L'impatience de cette génération «zapping» impose la rapidité d'accès et de téléchargement. Les différences de «publics » suivants les composantes de formation démontrent que si dans certain département une plate-forme numérique classique serait acceptable, pour d'autre formation, elle serait inévitablement vouée à l'échec. Si les machines aujourd'hui atteignent leur limite de performance, les réseaux quant à eux voient leur puissance doublée tous les neuf mois ${ }^{9}$. Ce qui nous amène à penser que SCOL est totalement adapté aujourd'hui à ce genre d'application.

Cette proposition de campus virtuel doit très prochainement être testé avec des étudiants du département GEII de l'IUT du Havre. Nous espérons remporter un grand succès, comme celui obtenu auprès des «adultes» testeurs. En effet, ce site a déjà été présenté en avril 2004 aux enseignants de ce département, qui ont eu des réactions très proches de celles attendues des apprenants (chat privé, chat public, utilisation d'un tableau blanc, branchement d'automates industriels via le campus virtuel et visualisation de résultats dynamiques,...). Nous avons pu constater que les comportements des enseignants de ce département ne sont pas si éloignés de ceux des étudiants, surtout quand il s'agit d'un environnement $3 \mathrm{D}$ qui fait appel à des souvenirs de jeux. Cette observation nous amène à la nécessité de responsabiliser les apprenants par :

* un filtrage par mot de passe sécurisé,

- une gestion sans ambiguïté des intervenants sur le site (Nom $\rightarrow$ mot de passe $\rightarrow$ avatar) et ce, grâce au SGBD,

* la présence d'un modérateur robotisé ou humain,

- un archivage de l'ensemble des actions pour une consultation éventuelle.

Cette approche du e-learning focalise sur le contenant et non sur le contenu. Le site, qu'il soit 3D ou non, doit être nourri des apports pédagogiques des collègues pour lesquels SCOL ne saurait être d'un grand secours.

\section{Bibliographie}

[1] 300 plates-formes e-formation, plates-formes elearning. Consulté le 25 mars 2005. Téléchargeable: http://thot.cursus.edu/rubrique.asp ?no $=12074$

[2] CARR, S. (2000). As distance education come of age, the challenge is keeping the students. The Chronicle of Higher Education, 46 (23), A39-A41.

[3] ISABELlE, C., VEZINA, N., FOURNIER, H., FONGEMIE, P. et LAVOIE, E., (2004), Un environnement 3D pour faciliter la formation en ligne. Technologies de l'Information et de la Connaissance dans l'Enseignement Supérieur et l'Industrie, Compiègne : Université de Technologie de Compiègne, 257-264.

Téléchargeable :

http://archive-edutice.ccsd.cnrs.fr/edutice-00000727

[4] Site du dictionnaire terminologique de l'Office Québécois de la langue Française.

Consultable :

http://www.granddictionnaire.com

[5] Glossaire de la FOAD (Formation Ouverte et A Distance).

Téléchargeable :

http://tice.education.fr

\footnotetext{
${ }^{9}$ Projet grille INRIA 2005.
} 